\title{
HIV-Test und Beratung auf Initiative des Arztes, der Ärztin
}

Das Bundesamt für Gesundheit empfiehlt, den HIV-Test in der ärztlichen Praxis nicht nur auf Wunsch des Patienten nach den Regeln des VCT (voluntary counselling and testing) durchzuführen. Schwangeren Frauen soll z.B. weiterhin routinemässig der HIV-Test vom Arzt, von der Ärztin vorgeschlagen werden, so wie es überall in Europa der Fall ist und vom Bundesamt für Gesundheit 2003 [1] und 2004 [2] empfohlen wurde. Daneben gibt es eine Reihe von Symptomen und Krankheitsbildern, bei denen an eine HIV-Infektion gedacht werden müsste und die den Arzt oder die Ärztin veranlassen sollen, einen HIVTest vorzuschlagen. Die vorliegenden Empfehlungen der Sektion Aids zur Testung auf Initiative des Arztes, der Ärztin entstanden in Zusammenarbeit mit der Fachkommission Klinik und Therapie des BAG.

\section{Einleitung}

Das Konzept provider initiated counselling and testing (PICT) wurde vor kurzem eingeführt und von den Gesundheitsorganisationen der UNO empfohlen. Es ergänzt das Konzept VCT (voluntary counselling and testing), bei dem die Initiative für den HIV-Test von der Klientin oder dem Klienten ausgeht [3]. Der wesentliche Unterschied zwischen diesen beiden Ansätzen besteht darin, dass beim PICT ein Arzt, eine Ärztin der Person, die ihn/sie (meist aus anderen Gründen) aufsucht, den Test vorschlägt. Grundsätzlich müssen beide Verfahren die Kriterien der drei C erfüllen: «consentment» (Einwilligung: kein Test ohne ausdrückliche, freiwillig erteilte Zustimmung), «confidentiality» (Vertraulichkeit: Test und Resultat für Dritte nicht zugänglich) und «counselling» (Beratung vor und nach dem Test).

\section{Warum sollen Ärztinnen und Ärzte mehr HIV-Tests vorschlagen?}

Es gibt eine Reihe von Situationen in bezug auf sexuelles Verhalten und/oder Symptome und Krankheitsbilder, die praktizierende Ärztinnen und Ärzte motivieren sollen, auch an HIV zu denken und von sich aus den HIV-Test vorzuschlagen. Insbesondere geht es um drei Ziele:

- Primärinfektionen nicht zu verpassen;

- bei Hinweisen auf riskantes Sexualverhalten in Umgebungen mit erhöhter Prävalenz den Test und vor allem das präventive Beratungsgespräch vorzuschlagen;
- bei unklaren Symptomen und Krankheitsbildern an eine fortgeschrittene HIV-Infektion zu denken.

\section{Der Verlauf der HIV-Infektion und der Virämie}

Die HIV-Infektion verläuft in drei Phasen (Abb. 1): 1. Nach einer Inkubationszeit von einigen Tagen bis einigen Wochen tritt bei der infizierten Person eine sehr ansteckende Primärinfektion auf, die sich als sehr heterogenes Syndrom präsentiert.

2. Darauf folgt eine asymptomatische Latenzzeit ohne wesentliche klinische Zeichen, die auf eine Infektion hinweisen (aber häufig eine periphere Lymphadenopathie).

3. Schliesslich treten bei einer fortgeschrittenen Infektion die sogenannt opportunistischen Erkrankungen auf, die in der Regel bei Personen mit intaktem Immunsystem nicht ausbrechen.

In jeder dieser Phasen kann sich der Leistungserbringer veranlasst sehen, einen HIV-Test vorzuschlagen. Er muss sich dabei auf die Kenntnis der Symptome stützen, die (in den Phasen 1 und 3) auf eine HIV-Infektion hinweisen. Ergänzend sind durch eine allgemeine Anamnese (Sexualpraktiken, intravenöser Drogenkonsum und Transfusionen) die Risiken abzuklären, denen sich die Patientin oder der Patient aussetzt und die einen HIV-Test rechtfertigen. Da der medizinische und verhaltensbezogene Nutzen der Ab- 
Abbildung 1

Verlauf der HIV-Infektion.

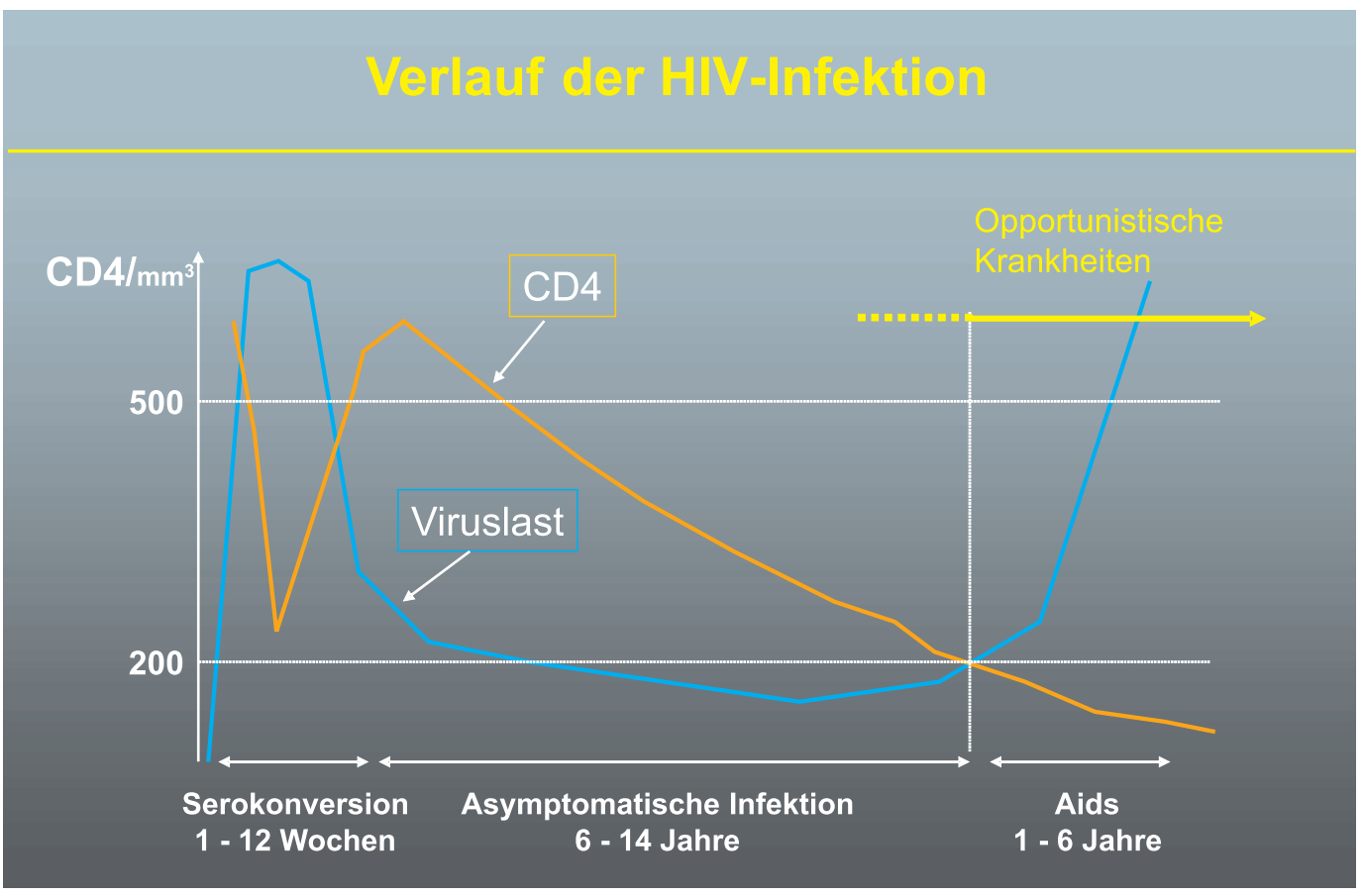

klärung des Serostatus im Verlauf der Infektion abnimmt, geht es darum, HIV in einem frühen Stadium zu erkennen: Während der akuten Infektion ist die Person besonders infektiös, und in dieser Phase wird das Virus am häufigsten übertragen [4].

\section{Situationen, in denen systematisch ein HIV-Test indiziert ist}

In den folgenden Fällen sollte systematisch ein HIV-Test empfohlen werden: Schwangerschaft, STI (Syphilis, Infektion mit N. gonorrhoeae, Chlamydia, Lymphogranuloma venereum, Herpes genitalis, Condylomata acuminata), Tuberkulose, Meningitis, Hepatitis (B und C), mukokutane Läsionen mit Candida albicans, Herpes zoster, persistierender Herpes simplex, periphere Lymphadenopathie, seborrhoische Dermatitis, orale Haarleukoplakie, Thrombozytopenie, Lymphopenie und Exantheme. Dasselbe gilt bei einer parenteralen Exposition am Arbeitsplatz.

Bei einem Syndrom, das einer Mononukleose gleicht, ist immer an eine HIV-Infektion zu denken: Bei jedem Bluttest auf Mononukleose ist deshalb auch ein HIV-Test indiziert.

4 Brenner BG, Roger M, Routy JP, Moisi D, Ntemgwa M, Matte $C_{\text {, }}$ et al. High rates of forward transmission events after acute/early HIV-1 infection. J Infect Dis. 2007;195:951-9.

5 Huynh Do P, Bricaire F. La primoinfection VIH, comment la reconnaître. Praxis. 2000;89:70-8.

\section{Symptome einer Primärinfektion}

Generell tritt bei der Primärinfektion ein Syndrom auf, das einer akuten Mononukleose gleicht [5]. Eine HIV-Infektion kann Krankheitsbilder mit den nachstehenden Symptomen (mit abnehmender Häufigkeit) verursachen.
Die häufigsten Symptome

- Fieber;

- Müdigkeit;

- disseminierte Adenopathien;

- erythematöse Pharyngitis;

- Hauteruptionen;

- Kopfschmerzen;

- aseptische Meningitis;

- reaktive Lymphozyten (als Laborbefund).

\section{Weniger häufig auftretende Symptome}

- Myalgien und Arthralgien;

- akute Diarrhoe;

- Übelkeit und Erbrechen.

\section{Seltenere Symptome}

- Hepatosplenomegalie;

- neurologische Manifestationen;

- orale und genitale Ulzera.

\section{Sexualanamnese}

Eine Sexualanamnese muss in allen drei Phasen der Infektion durchgeführt werden. In der Latenzzeit (Phase 2) ist sie jedoch für die Empfehlung des HIV-Tests ausschlaggebend. Denn liegen keine Symptome vor, ist die Sexualanamnese das einzige Instrument, das auf eine HIV-Infektion hinweisen kann.

Im Rahmen der Sexualanamnese sind die folgenden Themen anzusprechen:

- sexuelle Ausrichtung;

- ausgeübte Sexualpraktiken; 
- Verwendung von Präservativen;

- Partnerschaft: anonym, Gelegenheitspartner, feste Partnerschaft;

- Anzahl Partner;

- Häufigkeit von Geschlechtsverkehr;

- Alkohol-, Substanzkonsum (intravenöser Drogenkonsum);

- sexuelle Gewalt;

- Sextourismus.

Mit dieser Checkliste lassen sich die sexuellen Risiken einschätzen, die eine Person eingeht. Sie bildet gegebenenfalls die Grundlage für die Empfehlung eines HIV-Tests. Der Arzt, die Ärztin kann dem Patienten auch die Fragen im neuen Beratungstool www.check-your-lovelife.ch (online verfügbar) stellen und die Antworten des Patienten selbst eingeben. Die elektronische Auswertung am Ende der kurzen Befragung kann dem Arzt, der Ärztin die Vor- und Nachtestberatung erleichtern. Ein Ausdruck der Auswertung kann zur KG gelegt werden.

\section{Opportunistische Erkrankungen}

Auch nach der Latenzzeit lässt sich eine nichterkannte Seropositivität nicht ausschliessen. Opportunistische Erkrankungen sind meist keine Neuinfektionen, sondern entstehen durch Reaktivierung einer bereits vorliegenden «schlafenden» Infektion. Dabei handelt es sich um pulmonale, viszerale, neurologische, dermatologische, hämatologische und ophthalmologische Erkrankungen (sowie um HIV-assoziierte Malignome).

Entsprechend der abnehmenden CD4-Lymphozytenzahl $\left(<200 / \mathrm{mm}^{3}\right)$ treten vor allem die folgenden opportunistischen Erkrankungen auf:

- Tuberkulose;

- Soorösophagitis;

- Infektionen mit Pneumocystis jiroveci (früher carinii);

- persistierender Herpes simplex, Virus 1 und 2;

- Kaposi-Syndrom;

- Zervixkarzinom;

- Kryptosporidiose;

- Leukoenzephalopathie;

- Toxoplasmose;

- Non-Hodgkin-Lymphom;
- Kryptokokkose;

- Infektionen mit Zytomegalovirus;

- Infektionen mit atypischen Mykobakterien.

\section{Zusammenfassung}

1. Erkennen der Symptome der Primärinfektion und Nachweis mit angemessenen Tests (Phase der höchsten Infektiosität).

2. Systematische Empfehlung eines HIV-Tests: bei Schwangerschaft, STI, Tuberkulose, Mononukleose, mukokutanen Läsionen, Exanthemen, bei Vorliegen von Risikofaktoren in bezug auf das Sexualverhalten, bei intravenösem Drogenkonsum mit gemeinsamem Spritzengebrauch und bei Vorliegen von Transfusionen.

3. Liegen keine Symptome vor, wird empfohlen, sich auf die Resultate der Sexualanamnese abzustützen.

4. Auch an die Möglichkeit einer opportunistischen Erkrankung denken, um einen HIV-Test vorzuschlagen.

Das Bundesamt für Gesundheit empfiehlt allen Ärztinnen und Ärzten, den HIV-Test in den erläuterten Situationen von sich aus vorzuschlagen. Mehr richtig indizierte HIV-Tests auf Vorschlag der Ärzteschaft und Förderung des voluntary counselling and testing (VCT) bei den Zielgruppen der HIV-Prävention sollen sich ergänzen und helfen, die Zahl undiagnostizierter HIV-Infektionen in der Schweiz zu reduzieren. Ein Nutzen für die öffentliche Gesundheit entsteht aber nur, wenn einerseits Menschen, die sich wegen eines eingegangenen Risikos testen lassen wollten, auch adäquat beraten werden, damit sie ihr Verhalten ändern, und wenn andererseits mit Menschen, bei denen eine HIV-Infektion diagnostiziert wurde, auch primärpräventiv gearbeitet wird, insbesondere was ihre festen Partnerschaften betrifft. Die Fachkommission Klinik und Therapie erarbeitet zurzeit im Auftrag der Eidgenössischen Kommission für Aidsfragen (EKAF) Empfehlungen für die ärztliche Beratung von HIV-positiven Menschen. Mit deren Verabschiedung und Publikation ist noch vor den Sommerferien $\mathrm{zu}$ rechnen. 